\title{
Características y propiedades funcionales del maíz morado (Zea mays L.) var. subnigroviolaceo
}

\section{Characteristics and functional properties of purple corn (Zea mays L.) var. subnigroviolaceo}

\author{
Jhoseline Guillén-Sánchez, Sigry Mori-Arismendi; Luz María Paucar-Menacho* \\ Departamento de Ingeniería Agroindustrial, Facultad de Ingeniería, Universidad Nacional del Santa, Ancash-Perú.
}

Recibido 27 septiembre 2014. Aceptado 29 noviembre 2014.

\begin{abstract}
Resumen
El maíz morado es una planta oriunda de América, que tiene el epispermo de las semillas (granos) y la tusa (coronta) de color morado, lo que le otorga características especiales a los pigmentos que poseen (entre $1,5 \%$ y $6,0 \%$ ), llamados antocianinas, que pertenecen al grupo de los flavonoides. Debido a su alto contenido de antocianinas (cianin-3-glucosa $\mathrm{C} 3 \mathrm{G}$ que es su principal colorante) y compuestos fenólicos actúa como un poderoso antioxidante natural y anticancerígeno, teniendo además propiedades funcionales debido a estos compuestos bioactivos. El maíz morado además aporta cantidades importantes de almidón, cerca del $80 \%$; un $10 \%$ de azúcares los cuales le confieren un sabor dulce, un $11 \%$ de proteínas, $2 \%$ de minerales y vitaminas (complejo B y ácido ascórbico) concentrados en el endospermo. Además del valor nutricional, el maíz morado tiene una composición rica en fitoquímicos, que tienen efectos benéficos en nuestro cuerpo, tales como neutralizar los radicales libres y actuar como antimutagénico. Este trabajo de revisión tuvo como objetivo, recopilar información sobre los estudios realizados al maíz morado, como alternativa al uso de colorantes artificiales de alimentos y por sus beneficios a la salud al incluirlo en la dieta, tales como, enfermedades cardiovasculares (hipertensión arterial), reducción del colesterol, lucha contra la diabetes, siendo el más resaltante la acción antioxidante (antiarrugas).
\end{abstract}

Palabras clave: Antocianina, Flavonoide, Fitoquímico, Propiedades funcionales, Compuestos Bioactivos.

\begin{abstract}
Zea Mays L. variety purple (purple corn) is a plant native of America, which has the episperm seeds (grains) and the cobs (cob) in purple, which gives special characteristics to the pigments that have (between $1.5 \%$ and $6.0 \%$ ), called anthocyanins, which belong to the group of flavonoids. Due to its high content of anthocyanins (cianin C3G-3-glucose as its main color) and phenolic compounds act as powerful natural antioxidant and anticancer, Further having functional properties due to these bioactive compounds. The purple corn also provides significant amounts of starch, about $80 \%$ (complex carbohydrate), $10 \%$ of sugars provide sweetness, up to $11 \%$ protein, up to $2 \%$ minerals and B vitamins and ascorbic acid, concentrated in endosperm (grain free envelope). Also the nutritional value, the purple corn has a rich composition of phytochemicals, which have beneficial effects on our body, such as neutralize free radicals and act as antimutagenic. His review paper aimed to collect information on the studies undertaken to purple corn as an alternative to artificial food dyes and health benefits when included in the diet. Benefits such as cardiovascular diseases (hypertension), lowering cholesterol, fighting diabetes, being the most remarkable antioxidant action (wrinkle).
\end{abstract}

Keywords: Anthocyanin, Flavonoid, Phytochemical, Functional properties, Bioactive Compound.

\section{Introducción}

El Zea mays L. variedad morado (maíz morado) es un cereal oriundo del Perú y México, cuyas culturas precolombinas lo consideraron sagrado. Florece, cultivado o en estado silvestre, en diversos lugares de América. El maíz morado se cultivaba en el Perú en épocas prehispánicas y era conocido como oro, sara o kulli sara (Ortiz, 2013).

\footnotetext{
* Autor para correspondencia

E-mail: luzpaucar@uns.edu.pe (L. Paucar).
} 
El Zea mays L. variedad morado, es una variedad genética de maíz peruano; una mazorca (tusa y grano) constituido en un $85 \%$ por grano y $15 \%$ por coronta (tusa), este fruto contiene el pigmento denominado antocianina, que se encuentra en mayor cantidad en la coronta y en menor proporción en el pericarpio (cáscara) del grano, siendo uno de los principales alimentos en la dieta peruana, utilizado frecuentemente en la preparación de bebidas como la chicha morada y postres como la mazamorra morada (Otiniano, 2012).

Así, este trabajo tuvo como fin recoger información de los estudios realizados al maíz morado como colorante natural, sustituto de los colorantes químicos empleados en distintos alimentos, así como el impacto que tiene su consumo diario en la salud.

\section{Componentes benéficos del maíz Morado}

Los componentes químicos en el maíz morado son: ácido salicílico, grasas, resinas, saponinas, sales de potasio y sodio, azufre y fósforo, y sus compuestos fenólicos (Arroyo et al., 2010).

Los compuestos fenólicos contenidos en el maíz morado, actúan como antioxidantes, secuestrando especies reactivas de oxígeno e inhibiendo las enzimas productoras de radicales libres (Atmani et al., 2011).

Dentro de los compuestos fenólicos, tenemos a las antocianinas; concretamente, pigmentos hidrosolubles ampliamente distribuidos en el reino vegetal (Aguilera $e t$ al., 2011). Estas representan los principales pigmentos hidrosolubles visibles al ojo humano, debido al color púrpura que presentan. El color de las antocianinas depende de varios factores intrínsecos, como son los sustituyentes glicosídicos en las posiciones 3 y/o 5 con mono, di o trisacáridos y de acilación incrementando su solubilidad; demostrando que producen efectos en el tono de las antocianinas hacia las tonalidades púrpura y la posición de los mismos en el grupo flavilio; por ejemplo, si se aumentan los hidroxilos del anillo fenólico se intensifica el color azul, mientras que la introducción de metoxilos provoca la formación del color rojo (Aguilera et al., 2011).

En la planta de maíz, las antocianinas están presentes en diferentes estructuras, como tallo, vaina, hojas e inflorescencias; en la mazorca se pueden encontrar en cáscara y grano. En el grano se ha reportado la presencia de antocianinas principalmente en el pericarpio (Salinas et al., 2012).

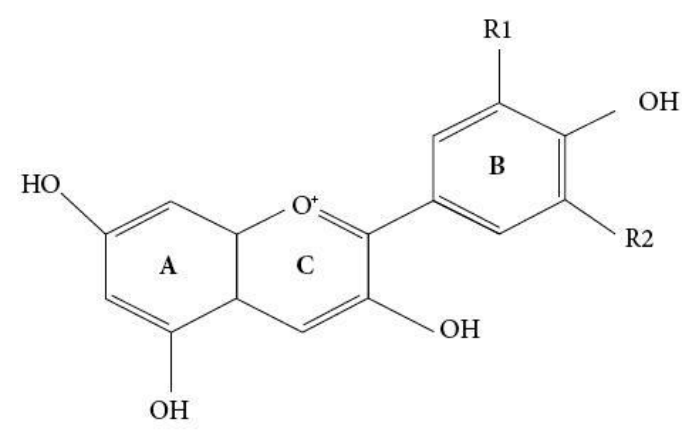

Figura 1. Estructura de las antocianinas en Frutos y Vegetales (de Pascual-Teresa y Sánchez-Ballesta, 2008).

Yolanda et al. (2013) reporta que la cáscara del maíz morado contiene aproximadamente 10 veces más antocianinas que otras plantas, siendo más frecuentes encontrarlas en flores y frutos, estas estructuras son las que contribuyen a los brillantes colores rojos, azules $\mathrm{y}$ morados de estos tejidos vegetales. Podemos anticipar la producción industrial de antocianina, porque la cáscara de maíz morado contiene $10 \%$ de antocianinas.

Recientes investigaciones informan sobre la existencia de cianidina 3 - glucósido en el grano del maíz morado, como la principal antocianina (flavonoide) contenida en este fruto. Otras antocianinas identificadas fueron cianidina 3-(6"malonil glucósido) y peonidina 3glucósido (Yolanda et al., 2013). La cianidina 3-glucósido, una importante antocianina presente en el maíz morado, suprime el 7,12-dimethilbenzo antraceno, 
el cual induce a la carcinogénesis mamaria, lo que indica que el color de maíz morado puede ser un agente quimioterapéutico prometedor (Fukamachi et al., 2008).

En este trabajo de revisión se consideran interesantes estos pigmentos por dos razones. La primera, por su impacto sobre las características sensoriales de los alimentos (de Pascual-Teresa y SánchezBallesta, 2008), las cuales pueden influenciar su comportamiento tecnológico durante el procesa-miento de alimentos; y la segunda, por su implicación en beneficio de la salud humana (Otiniano, 2012).

\section{Maíz morado como alimento funcional}

El maíz morado es un antioxidante natural que retarda el envejecimiento celular, principalmente por los mecanismos de acción de la cianidina-3- $\beta$-glucósido, pelargonidina-3- $\beta$-glucósido, peonidina-3$\beta$-glucósido, ácidos fenólicos, quercetina y hesperidina (Salinas et al., 2013). Varias investigaciones (Rachelle et al., 2014; Salinas et al., 2013; Bhornchai et al., 2014) validan sus propiedades farmacológicas, ya que contrarrestan los efectos nocivos de los radicales libres, estrés oxidativo y la carcinogénesis.

$\mathrm{Se}$ ha demostrado, que por su alto contenido en antocianinas tiene un efecto potenciador sobre la actividad de la superóxido dismutasa (SOD), en animales mono gástricos. Por tanto, su consumo también podría afectar el estado del sistema de defensa antioxidante en los rumiantes (Kenji et al., 2012).

Por otra parte, se ha confirmado que algunas de las antocianinas presentes en este alimento poseen efectos potenciadores sobre la expresión de ARNm y la actividad de superóxido dismutasa (SOD), que es una enzima antioxidante importante en los organismos vivos (Han et al., 2006).

$\mathrm{Se}$ ha informado de que los pequeños rumiantes como las ovejas y cabras pueden experimentar estrés oxidativo influenciada por la nutrición, estaciones climatológicas y su etapa fisiológica (Celi et al., 2010). Para hacer frente al daño oxidativo, la defensa antioxidante es administrado principalmente por las acciones de sustancias antioxidantes que neutralizan los radicales libres y enzimas que catalizan las reacciones de radicalización de peróxido (Jacob, 1995).

\section{Investigaciones científicas aplicadas en ratas de laboratorio}

Una investigación científica realizada por Pedreschi y Cisneros-Zevallos (2006), comprobó que las fracciones fenólicas obtenidas a partir de maíz morado tienen propiedades antimutagénicas. Este estudio evaluó si las antocianinas de maíz morado retardan la hiperglucemia crónica; el extracto de maíz morado rico en antocianinas suprimió la proliferación de células colorrectales en humanos y ejerce una interacción aditiva con los otros compuestos fenólicos funcionales (Jing y Giusti, 2007; Jing et al., 2008).

Arroyo et al. (2010) describieron el efecto hipotensor de un extracto de Zea mays L. (maíz morado) en ratas hipertensas. Esta reducción de la presión arterial fue explicada por la actividad vasodilatadora, dependiente de óxido nítrico, de dicho extracto. No obstante, diferentes extractos de maíz morado contienen diferentes moléculas adicionales (Arroyo et al., 2010) que podrían disminuir o incrementar el efecto vasodilatador de las antocianinas.

El mejoramiento de la agudeza visual y del comportamiento cognitivo como resultado del consumo de antocianinas ha sido reportado por Moreno-Loaiza (2013) donde se demostró que el comportamiento cognitivo y las funciones neuronales de ratas de laboratorio (Normas ISO $9000 /$ 9001 / 9002 / 9003) puede ser mejoradas a través de suplementación nutricional con extractos de arándanos y fresas que también contienen gran cantidad de antocianinas al igual que el maíz morado. Estos efectos sugieren que las antocianinas ostentan propiedades funcionales interesantes, y podría representar una promete- 
dora clase de compuestos útiles en el tratamiento de patologías.

\section{Benéfico contra la hipertensión}

A nivel mundial, las enfermedades cardiovasculares son responsables de aproximadamente 17 millones de muertes por año, casi un tercio del total. Se estima que la hipertensión es la causa de por lo menos el $45 \%$ de las muertes por cardiopatías en el planeta (World Health Organization, 2013); sólo en el Perú, esta enfermedad es uno de los principales problemas de salud pública; los resultados de una Encuesta Demográfica y de Salud Familiar del año 2012 mostraron que, un $29,7 \%$ de la población adulta mayor declaró haber sido informada en algún momento por un médico o profesional de la salud que padece de hipertensión arterial; un $70,1 \%$ seguían tratamiento antihipertensivo farmacológico y el 29,9\% a ningún tratamiento (INEI, 2012).

A pesar de los logros en el control de esta enfermedad por medidas preventivas como farmacológicas, se siguen buscando opciones para reducir las complicaciones asociadas con esta enfermedad.

\section{Beneficios como antioxidantes y los efectos de la antocianina}

Aunque hay varias investigaciones científicas sobre las propiedades biológicas de las antocianinas del maíz morado y su poder antioxidante in vitro, hay una falta de pruebas in vivo. El amplio conocimiento de la biodisponibilidad y el metabolismo de las antocianinas es, pues, esencial para que sus efectos sobre la salud sean entendidos. La evidencia actual en la literatura pobremente describe el metabolismo de las antocianinas en el ser humano, sin información sobre los efectos biológicos de sus metabolitos (Fernández et al., 2013).

Las antocianinas se han propuesto para ser absorbidas en el estómago (Fernández et al., 2012; Passamonti et al., 2003; Talavera et al., 2003) y el intestino delgado (Talavera et al., 2004) apareciendo en la circulación sanguínea y orina intactas y/o formas conjugadas (metilado, glucurono y/o formas sulfoconjugadas) (Felgines et al., 2003; Kay et al., 2005; Kay et al., 2004; Talavera et al., 2004; Wu et al., 2002). Sin embargo, la identificación de metabolitos derivados ha sido limitada como resultado de su diversidad, bajas concentraciones en la sangre y la falta de normas mundiales.

A diferencia de otros flavonoides, la síntesis química de los metabolitos de antocianinas es limitada debido a su baja estabilidad en su síntesis bajo condiciones ( $\mathrm{pH}$ y temperatura) normalmente aplicados a otros compuestos flavonoides que obtienen metabolitos (Dueñas et al., 2012). Sin embargo, algunos metabolitos de antocianina se han sintetizado enzimáticamente (Fernández et al., 2009). Como es fundamental determinar si estos nuevos compuestos son responsables de algunas actividades biológicas reportadas por las antocianinas, la purificación de metabolitos de antocianinas es crucial.

Recientemente, diversos materiales conteniendo antocianinas están siendo incorporados a productos alimenticios, donde tales productos requieren investigación a futuro para demostrar sus efectos fisiológicos.

\subsection{Como alimento sin preparar}

La mayonesa, una emulsión de aceite en agua $\left(\mathrm{A}_{\mathrm{C}} / \mathrm{A}_{\mathrm{G}}\right)$ que contiene de $70 \%$ a $80 \%$ grasa y yema de huevo (Chun et al., 2013), como todo alimento alto en grasa, es susceptible de deterioro debido a la auto oxidación que conduce a la reducción del almacenamiento, pero si le adicionamos antocianinas del maíz morado podrían potenciar un efecto antioxidante en esta mayonesa (PCHE "purple corn husk extract"), tal como lo demuestra investigaciones que comparan su efecto antioxidante en relación a los antioxidantes químicos BHT y EDTA (Chun et al., 2013). En esta investigación, mayonesa con $0,4 \mathrm{~g} / \mathrm{kg}$ PCHE mostró un mejor efecto antioxidante durante el almacenamiento. 
Este estudio sugiere que PCHE podría ser utilizado como antioxidante natural en alimentos con alto contenido de grasa y como sustituto de los antioxidantes químicos con su color violáceo que marca su diferencia con la mayonesa normal. Tal diferencia de color les dirá a los consumidores que su alimento contiene antioxidantes naturales.

Los productos en los que se emplean comercialmente las antocianinas como colorantes se incluyen principalmente los refrescos, dulces, caramelos, gomas de mascar, mermeladas, jaleas y conservas de frutas (Boo et al., 2012). Por tanto, la incorporación de antocianinas en los alimentos, además de ser benéficas para nuestra salud, mejora la apariencia total en los alimentos.

\subsection{Como alimento preparado}

En este caso, los tratamientos térmicos al que puede someterse el maíz morado pueden afectar en cierta parte su calidad nutricional. Así, Bhornchai et al. (2014) investigó los cambios de los componentes antioxidantes, incluyendo antocianinas y compuestos fenólicos, durante la cocción tradicional de dulce de maíz ceroso de color púrpura. Las mazorcas de maíz fueron sometidas a ebullición y cocidas al vapor debido a que estos son los métodos comunes de cocer el maíz.

Se pudo comprobar que el proceso de cocción tuvo un impacto significativo sobre la retención de contenido en antocianina monomérica. Cocinar por ebullición dio lugar a mayores descensos $(60,7 \%)$, seguido por granos enteros hervidos $(31,7 \%)$, granos cortados cocidos al vapor $(19,2 \%)$, y los granos enteros cocidos a vapor $(3,5 \%)$. Por otro lado, el efecto de la cocción de batatas reducía el contenido de antocianinas por casi la mitad de cantidad original (Kim et al., 2012).

La estabilidad de antocianinas y otros pigmentos en los alimentos disminuirá con el aumento de temperatura (Xu y Chang, 2008). Jing y Giusti (2007) observaron una disminución consistente de proteínas a 100
${ }^{\circ} \mathrm{C}$ en extractos de agua de maíz morado que indica una posible desnaturalización de proteína por las altas temperaturas, lo que podría resultar por la precipitación de la antocianina que conduce a una disminución de su contenido en las comidas preparadas. Por lo tanto, los diversos resultados indican la importancia del método de cocción en la retención de nutrientes y la preservación de los pigmentos naturales después del procesamiento térmico, los cuales son importantes parámetros de calidad. Finalmente, las propiedades funcionales de las antocianinas y su manera de consumirlas abren una nueva perspectiva para la obtención de productos coloreados con valor agregado para el consumo humano.

\section{Conclusiones}

En este trabajo se ha definido al maíz morado como el mejor sustituto para colorantes alimenticios artificiales, debido a su origen natural y alto contenido en antioxidantes. Las investigaciones científicas demuestran que los pigmentos hidrosolubles presentes en el maíz morado denominados antocianinas, pueden utilizarse en las industrias farmacológicas y alimentarias con la finalidad de obtener un producto funcional en beneficio de la salud de sus consumidores otorgándole un alto valor agregado. Estudios experimentales han demostrado que el incremento en el consumo de antocianinas puede disminuir la presión sanguínea en personas hipertensas y elevar la capacidad antioxidante total de la sangre. Asimismo, su ingestión regular podría ser útil para personas que no padecen de ninguna enfermedad ya que pueden mejorar su estilo de vida, debido a que no presenta efectos secundarios. Las propiedades que posee el maíz morado son poco conocidas por quienes lo consumen pero investigaciones futuras (infusiones de antocianina o aceite de maíz morado) y una adecuada información dará a conocer las facultades que posee, lo cual motivaría su consumo intensivo y permanente. 


\section{Referencias bibliográficas}

Aguilera, M.; Reza, M.; Chew, R.; Meza, J. 2011. Propiedades funcionales de las antocianinas. Revista de ciencias biológicas y de la salud 13: 16-22.

Arroyo, J.; Saez, E.; Rodríguez, M.; Chumpitaz, V.; Burga, J.; de la Cruz, W.; Valencia, J. 2010. Reducción del colesterol y aumento de la capacidad antioxidante por el consumo crónico de maíz morado (Zea mays L.) en ratas hipercolesterolémicas. Revista Peruana de Medicina Experimental y Salud Publica 24: 157-162.

Atmani, D.; Ruiz-Larrea M.B.; Ruiz-Sanz, J.I.; Lizcano, L.J.; Bakkali. F.; Atmani, D. 2011. Antioxidant potential, cytotoxic activity and phenolic content of Clematis flammula leaf extracts. J. Med. Plants Res. 5: 589-598.

Bhornchai,H.; Bhalang, S.; Ratchada, T.; Scott, M.; Kamol, L. 2014. Anthocyanin, phenolics and antioxidant activity changes in purple waxy corn as affected by traditional cooking. Food Chemistry 164, 510-517.

Boo, H.; Hwang, S.; Bae, C.; Park, S.; Heo B.; Gorinstein S. 2012. Extraction and characterization of some natural plant pigments. Industrial Crops and Products. 40, 129-135.

Celi, P.; di Trana A.; Claps, S. 2010. Effects of plane of nutrition on oxidative stress in goats during the peripartum period. Vet. J. 184, 95-99.

Chun, Y.; Hee,W.; He, Li.; Deug, C.; Hae, I. 2013 Antioxidative effect of purple corn extracts during storage of mayonnaise. Food Chemistry 152: 592-596.

de Pascual - Teresa S.; Sánchez - Ballesta. 2008. Anthocyanin's: from plant to health. Phytochem. 7: 281-299.

Dueñas, M.; González-Manzano, S.; Surco-Laos, F.; González-Paramas, A.; Santos-Buelga, C. 2012. Characterization of sulphated quercetin and epicatechin metabolites. Journal of Agricultural and Food Chemistry 60: 3592-3598.

Felgines, C.; Talavera, S.; Gonthier, M.; Texier, O.; Scalbert, A.; Lamaison, J. et al. 2003. Strawberry anthocyanins are recovered in urine as glucuro and sulphoconjugates in humans. The Journal of Nutrition 133: 1296-1301.

Fernández, I.; Azevedo, J.; Faria, A.; Calhau, C.; de Freitas, V.; Mateus, N. 2009. Enzymatic hemisynthesis of metabolites and conjugates of anthocyanins. Journal of Agricultural and Food Chemistry 57: 735-745.

Fernández, I.; De Freitas, V.; Reis, C.; Mateus, N. 2012. A new approach on the gastric absorption of anthocyanins. Food \& Function 3: 508-516.

Fernández, I.; De Freitas, V.; Reis, C.; Mateus, N. 2013. Antioxidant and antiproliferative properties of methylated metabolites of anthocyanins. Food Chemistry 141: 2923-2933.

Fukamachi, K.; Imada, T.; Ohshima, Y.; Xu J.; Tsuda, H. 2008. Purple corn color suppresses Rasprotein level and inhibits 7,12-dimethylbenz[a]anthracene-induced mammarycarcinogenesis in the rat. Cancer Sci. 99: 1841-1846.

Han, K.; Sekikawa, M.; Shimada, K.; Hashimoto, M.; Hashimoto, N.; Noda, T.; Tanaka, H.; Fukushima, M.; 2006. Anthocyanin rich purple potato flake extract has antioxidant capacity and improves antioxidant potential in rats. Br. J. Nutr. 96: 1125-1133.

INEI - Instituto Nacional de Estadística e Informática. 2012. Condiciones de riesgo cardiovasculares. Perú: situación de salud de la población adulta mayor. $4 \mathrm{p}$.

Jacob, R. 1995. The integrated antioxidant system. Nutr. Res. 15: 755-766.
Jing P.; Giusti M. 2007. Effects of extraction conditions on improving the yield and quality of an anthocyanin-rich purple corn (Zea mays L.) color extract. Food Chem Toxicol. 72: 366-368.

Jing, P.; Bomser J.; Schwartz, S.; He, J.; Magnuson, B.; Giusti, M. 2008. Structure function relationships of anthocyanins from various anthocyanin-rich extracts on the inhibition of colon cancer cell growth. J Agric Food Chem. 56: 9391-9398.

Jing, Li.; Soon, L.; Jae-Yong, L.; Jin-Kyu, K.; Sang-Wook, K.; Jung-Lye, K.; Young-Hee, K. 2012. Purple corn anthocyanins dampened high glucose induced mesangial fibrosis and inflammation: possible renoprotective role in diabetic. J Nutr. Biochem. 23: 320331.

Kay, C.; Mazza, G.; Holub, B.; Wang, J. 2004. Anthocyanin metabolites in human urine and serum. British Journal of Nutrition 91: 933-942.

Kay, C.; Mazza, G.; Holub, B. 2005. Anthocyanins exist in the circulation primarily as metabolites in adult men. The Journal of Nutrition 135: 2582-2588.

Kenji, H.; Makoto, M.; Hiroki, M.; Satoshi, H.; Hiroshi, I.; Kazuhisa, N. 2012. Effect of supplementation of purple pigment from anthocyanin rich corn (Zea mays L.) on blood antioxidant activity and oxidation resistance in sheep. Livestock Science 145: 266-270.

Kim, H.W.; Kim, J.B.; Chao, S.M.; Chung, M.N.; Lee, Y.M.; Chu, S.M.; et al. 2012. Anthocyanin changes in the Korean purple-fleshed sweet potato, Shinzami, as affected by steaming and baking. Food Chemistry 130: 966-972.

Moreno-Loaiza, O.; Paz-Aliaga, A.; Mamani-Choquepata, P.; Mamani-Quispe, V.; Manchego-Rosado, L. 2013. Curva dosis-efecto de las antocianinas de tres extractos de Zea mays L. (maíz morado) en la vasodilatación de anillos aórticos de rata. Rev. Perú. Med. Exp. Salud publica 30: 714-728

Ortiz, K. 2013. Elaboración de un sorbete a base de harina de maíz morado (Zea mays L) mezclado con bacterias lácteas naturales. Universidad Dr. José Matías Delgado. El Salvador. Capítulo VI, Art. 46.

Otiniano, V. 2012. Actividad antioxidante de antocianinas presentes en la coronta y grano de maíz (Zea mays L.) variedad morada nativa cultivada en la ciudad de Trujillo. Tesis para optar el título de Ingeniero Agroindustrial, Universidad Cesar Vallejo. 74 p.

Passamonti, S.; Vrhovsek, U.; Vanzo, A.; Mattivi, F. 2003. The stomach as a site for anthocyanins absorption from food. FEBS Letters 544: 210-213.

Pedreschi R.; Cisneros-Zevallos L. 2006. Antimutagenic and antioxidant properties of phenolic fractions from Andean purple corn (Zea mays L.). J Agric. Food Chemical 54: 4557-4567.

Rachelle, M.; Esperance, D.; Rachad, S.; Richard, G.; Nicolas, L. 2014. Multiple optimizations of chemical and textural properties of roasted expanded purple maize using response surface methodology. Journal of Cereal Science 60: 397-405.

Salinas, Y.; García, C.; Coutiño, B.; Vidal, V. 2013. Variabilidad en contenido y tipos de antocianinas en granos de color azul/morado de poblaciones mexicanas de maíz. Rev. Fitotec. Mex. 285 -294.

Talavera, S.; Felgines, C.; Texier, O.; Besson, C.; Lamaison, J.; Remesy, C. 2003. Anthocyanins are efficiently absorbed from the stomach in anesthetized rats. The Journal of Nutrition 133: 4178-4182.

Talavera, S.; Felgines, C.; Texier, O.; Besson, C.; Manach, C.; Lamaison, J. et al. 2004. Anthocyanins are efficiently absorbed from the small intestine in rats. The Journal of Nutrition 134: 2275-2279. 
Wu, X.; Cao, G.; Prior, R. 2002. Absorption and metabolism of anthocyanins in elderly women after consumption of elderberry or blueberry. The Journal of Nutrition 132: 1865-1871.

World Health Organization. 2013. Global health risks: mortality and burden of disease attributable to selected major risks Geneva: World Health Organization, Organización Mundial de la Salud.
Xu, B.; Chang, S.K.C. 2008. Total phenolics, phenolic acids, isoflavones, and anthocyanin and antioxidant properties of yellow and black soybeans as affected by thermal processing. Journal of Agricultural and Food Chemistry 56: 7165-7175. 